\title{
Improved Ant Colony based Optimization based Lane Colorization for Curved Lane Images
}

\author{
Ritika \\ BTech, CSE \\ Computer Science \& Engg Department \\ Guru Nanak Dev University, Amritsar
}

\author{
Saransh Bhalla \\ BTech, CSE \\ Computer Science \& Engg Department \\ Guru Nanak Dev University, Amritsar
}

\begin{abstract}
There are many researchers who have worked and are working on creating and developing many techniques in intelligent transportation systems with advanced driving assistances system which are able to ensure the safety in the roads and congested traffic conditions. The road accidents are the main causes for the sudden death in this world. Even though we have many good and advanced techniques in this world, we are left over with something to make it better than before. In this paper, a new technique for lane detection using fuzzy $\mathrm{c}$ means clustering and ant colony optimization has been devised. The algorithm performs very efficiently in case of the curved roads.
\end{abstract}

\section{Keywords}

Lane Detection, ACO, Fuzzy C Means Clustering

\section{INTRODUCTION}

Everybody in this world is concerned about safety. The people those who go out from one place to other, expect to reach safely. Without any sudden incidents which may come through externally by road accidents while travelling. We can avoid the road accidents by using improved driving assistances. Vehicle crashes remain the leading cause of accidental death and injuries in most traffic congested countries e.g. UK, USA, and Asian countries claiming tens of thousands of lives and injuring millions of people each year. Most of these transportation deaths and injuries occur on the nation's highways. Therefore, a system that provides a means of warning the driver to the danger has the potential to save a considerable number of lives. One of the main technologies involves in these tasks is computer vision, which become a powerful tool for sensing the environment and has been widely used in much application by the intelligent transportation systems (ITS)[1].

In order to increase safety and reducing road accidents, people are spending lots of money for the advancement in the driving techniques which ensures the safety. The technology makes man to think more to improve the safety to save the lives. The automobiles are more conscious of providing safety feathers like seat belts, air bags and strong body structures which provide the passive safety that may reduce the effects of an accident. Avoiding accidents and saving lives are one of great interest that all researchers and Automobile companies work on. In Advanced Driver Assistance Systems in order to achieve the desired safety on roads, the complex and challenging tasks of future road vehicles are road lanes detection or boundaries detection (white and black lines on roads) and Obstacles detection (cars, pedestrians, trees, etc) especially for moving object detection is a key component of collision avoidance in driving assistance systems.
Many sensors can be used for lane detection and obstacle detection, such as laser, radar and vision sensors. Detecting all kinds of obstacles on the roads mainly include IPM (inverse perspective mapping) method. The system acquires the front view using a camera mounted on the vehicle then applying few processes in order to detect the lanes and objects. A versatile methodology is used in order to detect the lanes and objects. Cars equipped with intelligent system like road lane detection and obstacle detection makes vehicles safer, which is vital in decrease number of victims or injured people by car accidents. Principal approaches to detection are using vision system on the vehicle.

Lane detection is one of the methods which use the principle of vision based lane detection. As the name itself indicates is a process of detecting as well as recognizing the lanes where the ground traffic circulates. For driving advanced driving assistances the lane detection is one of the essential functions. The lane detection has become very specific term that implies the utilization of certain perceptive sensors, certain processing units, and certain algorithms to perform this functionality.

The required properties of lane detection techniques include: 1) Quality of lane detection shouldn't be afflicted with shadows, which may be cast by trees, buildings, etc.

2) It will manage to handle the curved roads as opposed to assuming that the roads are straight.

3) It might assume the parallelism of both sides of the lane markings to enhance the detection in the existence of noises in the images.

4) It will offer an explicit reliable measurement of the obtained result that individuals could know whether to abandon the method.

An excellent lane detection algorithm should satisfy most of the properties mentioned above.

\section{LITERATURE SURVEY}

Dietmayer, Klaus et al. [1] discussed that multilayer laser scanner sensors measure an exact range profile of road traffic conditions. Furthermore, they can detect different reflectivities of object surfaces. Along with object detection and tracking, sensory features facilitate a roadway and lane detection function devoid of requiring another sensors. The unique multilayer laser scanner prototype has been developed because of this purpose. The roadway detection is dependent on an identification of changes in the exact distance profiles pattern at the limits of roadways. The tracking and classification of reflection posts extends the effectual selection of the roadway detection and enhances its robustness. Lane detection utilizes the reflectivity of lane markings and surface of the road. The detection of lane 
operates inside a distance selection of four meters as much as thirty meters and has been tested on highway scenarios. Rotaru, C. et al. [2] presented different methods that treat the particular cases that can be found in the detection of lane marking in construction areas for both highways and country roads. The device complements the lane marking detection methods by treating the special case of temporary yellow markings that override the conventional white markings. It uses both position and color to split up the valid markings from the former ones left in position but without semantics for the driver. Zhou, Yong et al. [3] proposed an effective lane detection approach, that's primary and required for driver assistance systems, to take care of the situations where in actuality the lane boundaries in a graphic have relatively weak local contrast, or where there are strong distracting edges. The proposed lane detection approach utilizes a deformable template model to the expected lane boundaries in the image, a maximum a posteriori (MAP) formulation of the lane detection problem, and a particle swarm optimization algorithm to maximise the posterior density. The model parameters completely determine the career of the automobile within the lane, its heading direction, and the neighborhood structure of the lane. Experimental results demonstrate that the proposed method is robust against noise and shadows in the captured road images. Möhler, Nikolaus et al. [4] developed the goal of the SAFELANE project a situation-adaptive system for enhanced lane keeping support. A prerequisite for lane keeping is reliable information of the automobile environment. Especially the automobile position within the lane and the span of the trail ahead is important. These details are supplied by the lane detection component. The lane tracker is principally vision based however it can also be supplemented by map, positioning and vehicle data. A higher dynamic range camera offers the processing unit with image data. Measurement points of lane borders, calculated by a powerful edge detection algorithm, are accustomed to estimate a 3D clothoid type of the lane. A new approach to get an original lane model is introduced which is often dynamically adapted from map and positioning data. The detection is extended to the neighbour lanes and a lane markings type classifying component is added. Jiang, Ruyi et al. [5] introduced a new lane model in correspondence to the particle filter-based approach, which can be flexible to detect all sorts of lanes. A revised version of an Euclidean distance transform is put on an edge map of a road image from the birds-eye view to supply information for boundary point detection. An efficient lane tracking method can also be discussed. The utilization of this distance transform exploits useful information in lane detection situations, and greatly facilitates the initialization of the particle filter, in addition to lane tracking. Finally, the paper validates the algorithm with experimental evidence for lane detection and tracking. Jung, H. G et al. [6] discussed industry trends and features of a security system integrating LKS (Lane Keeping System) and ACC, known as the LKS+ACC system, and proposes a technique utilising the range data from ACC for the sake of lane detection. The entire structure of lane detection is just like the standard method using monocular vision: EDF (Edge Distribution Function)based initialization, sub-ROI for left/right and distance-based layers, steerable filter-based feature extraction, and model fitting in each sub-ROI. The proposed method adds only the device for confining lane detection ROI to free space that's established by range data. Experimental results indicate that this kind of simple adaptive ROI can overcome occlusion of lane markings and disturbance of neighboring vehicles. Romdhane et al. [7] proposed a lane detection method through digital image processing. Morphological filtering, Hough transform and linear parabolic fitting are placed on realize this task. The outcome of these proposed method are in contrast to three proposed researches. The technique presented here was tested on video sequences filmed by the authors on Tunisian roads, on a movie sequence supplied by Daimler AG in addition to on the PETS2001 dataset supplied by the ESSEX University. Ruyi, Jiang et al. [8] introduced a new weak lane model with this particular particle filter-based approach. This new model parameterizes the connection between points of left and right lane boundaries, and may be used to detect all kinds of lanes. Furthermore, an altered version of an Euclidean distance transform is applied on a benefit map to supply information for boundary point detection. When compared with a benefit map, properties with this distance transform support improved lane detection, including a new initialization and tracking method. This paper fully explains how the application form of the distance transform greatly facilitates lane detection and tracking. Two lane tracking methods may also be discussed while concentrating on efficiency and robustness, respectively. Finally, the paper reports about experiments on lane detection and tracking, and comparisons with other methods. Liu, Lizhuang et al. [9] tried to enhance the approach for the robustness. Their main idea may be the noise reduction centered on narrowing a size of search area. The proposed method uses the road model on the basis of the generalized hough transformation. They used the gradient direction to lessen the amount of votes and the strategy is Kernel-based Hough transform. It's shown that the technique can perform better detection result. Choi, H-C. et al. [10] proposed a vision-based fusion technique for lane tracking and forward vehicle detection to deal with challenging conditions, i.e., lane occlusion by way of a forward vehicle, lane change, varying illumination, road traffic signs, and pitch motion, which often occur in real driving environments. First, their algorithm uses random sample consensus (RANSAC) and Kalman filtering to calculate the lane equation from the lane candidates found by template matching. Simple template matching and a mix of RANSAC and Kalman filtering makes calculating the lane equation as a hyperbola pair very quick and robust against varying illumination and discontinuities in the lane. Second, their algorithm works on the state transfer technique to keep lane tracking continuously regardless of the lane changing situation. This reduces the computational time when working with the lane change because lane detection, which takes a great deal more time than lane tracking, isn't necessary with this specific algorithm. Third, false lane candidates from occlusions by frontal vehicles are eliminated using accurate elements of the forward vehicles from their improved forward vehicle detector. Fourth, their proposed method achieved robustness against road traffic signs and pitch motion utilizing the adaptive region of interest and a constraint on the positioning of the vanishing point. Their algorithm was tested with image sequences from a genuine driving situation and demonstrated its robustness. Yao, Wentao, and Zhidong Deng [11] proposed a construction of robust and real-time lane marking detection and tracking for the autonomous driving of intelligent vehicle under urban road environments. Their framework use hyperbola model because the lane marking model and several adaptive techniques to robustly extract lane marking pairs for the present lane. The framework supplies a complete solution from environment perception of intelligent vehicle to vehicle control. The experimental results achieved on urban road scenarios reveal that their system performs well under various situation. Moreira, Bruno $\mathrm{M}$ et al. [12] proposed a technique for automating the detection of 
lanes. Their approach features a preprocessing step to detect the image region of interest, accompanied by background estimation and removal. This image is then projected onto the horizontal direction to integrate the data right into a onedimensional profile. A smoothing filter is placed on this profile and the results could be the input of the lane detection process, which is conducted in three phases. The initial one aims at obtaining an original pair of candidate lanes which can be further validated or removed in the next phase. The past phase is really a refinement step which allows the inclusion of lanes which are not clearly distinguishable in the profile and which were not contained in the initial set. The technique was evaluated in 66 chromatography images and achieved values of recall, precision and $\mathrm{F}_{\beta}$-measure of $97.0 \%, 99.4 \%$ and $98.2 \%$, respectively. Liu, Weirong, and Shutao Li [13] proposed a highly effective and robust algorithm for structured road in urban. Firstly, the adaptive segmentation method can be used to ascertain reasonable ROI that covers all candidate lane markings. Secondly, line segments are extracted by Line Segment Detector (LSD) and be utilized as low level feature to extract the structural information of road scene. Thirdly, non-lane markings are eliminated by clustering on orientation information and vanishing point. Finally, the lanes are extracted from the rest of the candidate lane markings. Experimental results on Caltech lane datasets reveal that the algorithm can extract lanes in complex structured road scenarios. Tao, Junli et al. [14] contributed to the detection of driving on the incorrect side of the road by addressing specifically multi-lane road situations. They suggested a remedy using video data of an individual camera limited to identifying the existing lane of the ego-vehicle. GPS data are employed for knowing defined constraints on driving directions for the existing road. Kawatsu, Christopher et al. [15] described approach in developing a reasonable stereo vision system using two ordinary webcams and OpenCV library.. Bottazzi, Vitor S. et al. [15] proposed a vision-based ego-lane detection system with \{the capability|the ability|the capacity $\}$ of automatically adapting to abrupt lighting changes. The proposed method automatically adjusts the feature extraction and salient point tracking cues introduced by the GOLDIE algorithm. The variance of the lighting conditions is measured using huesaturation histogram and abrupt light changes traveling are detected on the basis of the difference between histograms. Experimental comparison with previously proposed algorithms demonstrated that this technique achieved efficient lane detection in the clear presence of shadows and headlights. Specifically, the accuracy of the algorithm applied on the footage with highest light variation increased $12.5 \%$ on average. The general detection rate increased $4 \%$, which illustrated the applicability of the method. Hillel, Aharon Bar et al. [16] surveyed the approaches and the algorithmic techniques devised for the different modalities throughout the last 5 years. They presented an universal breakdown of the situation into its functional blocks and elaborate the wide variety of proposed methods in this scheme. For every functional block, they described the possible implementations suggested and analyze their underlying assumptions. While impressive advancements were demonstrated at limited scenarios, inspection into the requirements of next generation systems reveals significant gaps. They identified these gaps and suggest research directions that could bridge them. Ran, Feng et al. [17] proposed an algorithm that assumed that lanes are usually the straight lines and whole algorithm is dependant on Hough transform. Because of the complexity of urban traffic scenes, false lane detections are highly due to warning lines and signs whose shapes and colors are like the lane boundary. In this study, they improved the accuracy of the lane detection base on Hough, a score function on the basis of the width between left and right lanes is proposed to acquire reliable lane detect results on urban traffic scene. Meanwhile, a listing of candidate lanes is constructed at least of execution time. Experiments under various scenes indicated that the proposed lane detection method can perhaps work robustly in the real-time. Bottazzi, Vitor S. et al. [18] discussed the adoption of an efficient model, which could monitor the ego-lane boundaries while identifying false positive references. The proposed architecture allows the mixture of multiple image-processing cues to enhance accuracy.

\section{PROPOSED METHODOLOGY}

Figure 1 represents the flowchart of the proposed methodology.

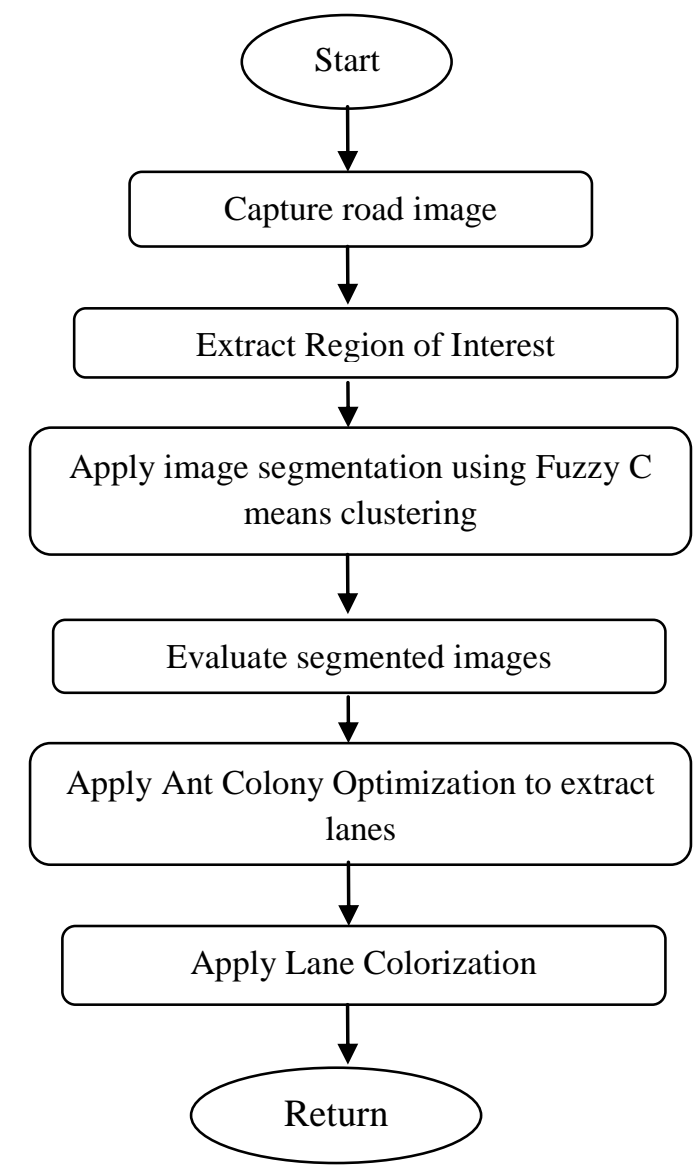

Fig 1: Flowchart of the proposed methodology.

\section{Steps of the proposed methodology}

Step 1: Take the road image.

Step 2: Extract region of interest of the road image.

Step 3: Then apply image segmentation using Fuzzy C means clustering

Step 4: In this step, the evaluation of segmented images is done.

Step 5: Here Ant Colony Optimization is applied to extract lanes.

Step 6: Here lane colorization is applied. 


\section{RESULTS AND DISCUSSIONS}

The proposed algorithm is tested on various images. The algorithm is applied using various performance indices accuracy, bit error rate, f-measure and specificity. In order to implement the proposed algorithm, design and implementation has been done in MATLAB using image processing toolbox. We are comparing proposed approach using some performance metrics

\section{EXPERIMENTAL SETUP}

Figure 2 represents the input road image and the results of the existing technique on input road image. From the figure it is clear that existing technique fails in case of curved roads.
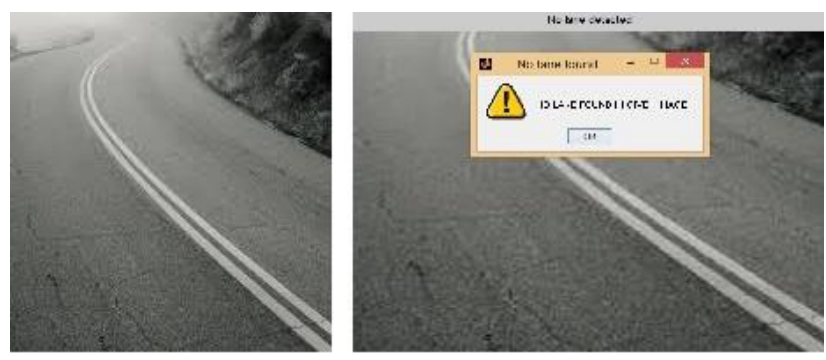

Fig 2 : Input Image and Results of Existing Technique

Figure 3 represents the input road image and the results of the proposed technique on input road image. From the figure it is clear that proposed technique gives efficient results in case of curved roads.

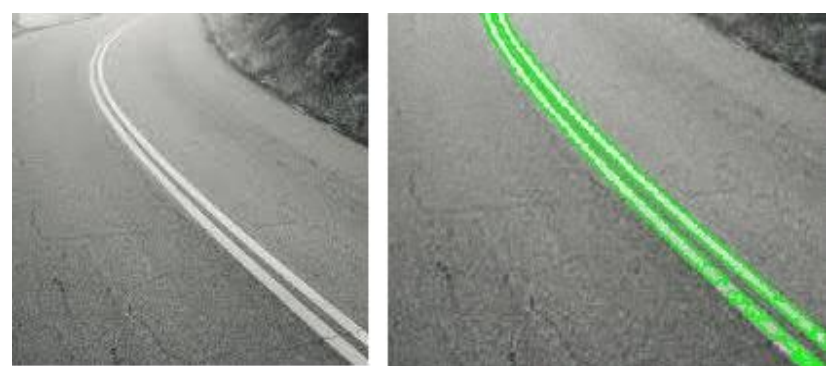

Fig 3: Input Image and Results of Proposed Technique

\section{PERFORMANCE EVALUATION}

\subsection{MSE}

In image processing mean square error is the most general measure for performance measurement of the existing method and the coded images. It is computed by using equation

$$
\operatorname{MSE}=\frac{1}{M N} \sum_{i=1}^{M} \sum_{j=1}^{N}\left(f(i, j)-f^{\prime}(i, j)\right)^{2}
$$

Table 1 has shown the analysis of the mean square error. Mean Square Error needs to be less for the proposed algorithm for obtaining better results than existing technique. As shown in the table the results for proposed algorithm are less in every case. This shows the efficiency of proposed algorithm.

Table 1: MSE Evaluation

\begin{tabular}{|l|l|l|}
\hline Image & $\begin{array}{l}\text { Existing } \\
\text { Technique }\end{array}$ & $\begin{array}{l}\text { Proposed } \\
\text { Technique }\end{array}$ \\
\hline 1 & 0.0102 & 0.0087 \\
\hline 2 & 0.0162 & 0.0004 \\
\hline 3 & 0.0127 & 0.0015 \\
\hline 4 & 0.0367 & 0.0235 \\
\hline
\end{tabular}

\begin{tabular}{|l|l|l|}
\hline 5 & 0.0117 & 0.0034 \\
\hline 6 & 0.0096 & 0.0043 \\
\hline 7 & 0.0239 & 0.0028 \\
\hline 8 & 0.0100 & 0.0061 \\
\hline 9 & 0.0115 & 0.0034 \\
\hline 10 & 0.0422 & 0.0078 \\
\hline
\end{tabular}

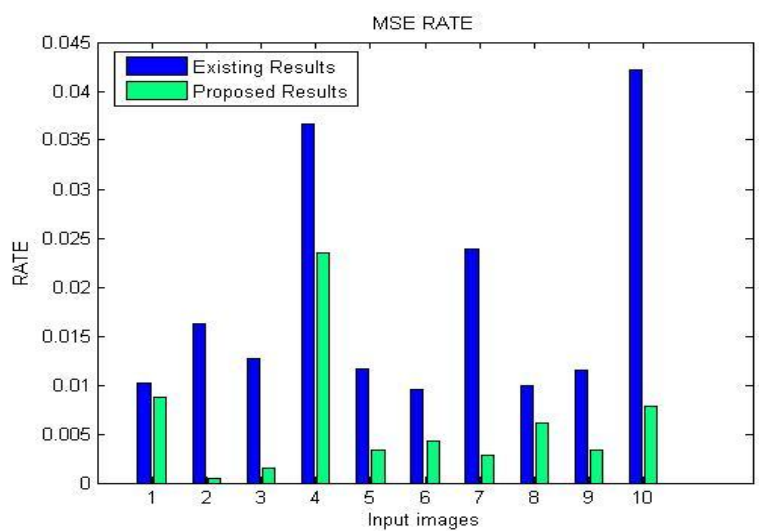

Fig 4: MSE Analysis

Figure 4 is showing the comparative analysis of the Mean Square Error. Mean Square Error needs to be less for the proposed algorithm for obtaining better results than existing technique. It can be seen clearly in the graph above, the results for proposed algorithm are less in every case as compared to existing technique. This shows the efficiency of proposed algorithm.

\subsection{PSNR}

The PSNR block computes the peak signal-to-noise ratio, between two images. This ratio is often used as a quality measurement between the original and a fused image. The higher the PSNR shows the better the quality of the fused or reconstructed image. PSNR value is computed by following equation:

$$
P S N R=10 \log _{10}\left(\frac{255^{2}}{M S E}\right)
$$

As PSNR need to be maximized; so the main goal is to increase the PSNR as much as possible.

Table 2 shows the comparable analysis of the Peak Signal to Noise Ratio (PSNR). PSNR needs to maximum for the proposed algorithm than existing techniques. As shown in the table the results for proposed algorithm are maximum in every case. Therefore proposed algorithm is providing better results than existing techniques.

Table 2 :PSNR Evaluation

\begin{tabular}{|l|l|l|}
\hline Image & $\begin{array}{l}\text { Existing } \\
\text { Technique }\end{array}$ & $\begin{array}{l}\text { Proposed } \\
\text { Technique }\end{array}$ \\
\hline 1 & 17.31 & 20.40 \\
\hline 2 & 17.89 & 19.93 \\
\hline 3 & 9.88 & 11.19 \\
\hline 4 & 13.41 & 15.05 \\
\hline 5 & 15.32 & 17.18 \\
\hline 6 & 12.78 & 13.66 \\
\hline
\end{tabular}




\begin{tabular}{|l|l|l|}
\hline 7 & 17.59 & 19.56 \\
\hline 8 & 13.16 & 13.95 \\
\hline 9 & 20.65 & 23.03 \\
\hline 10 & 16.00 & 18.40 \\
\hline
\end{tabular}

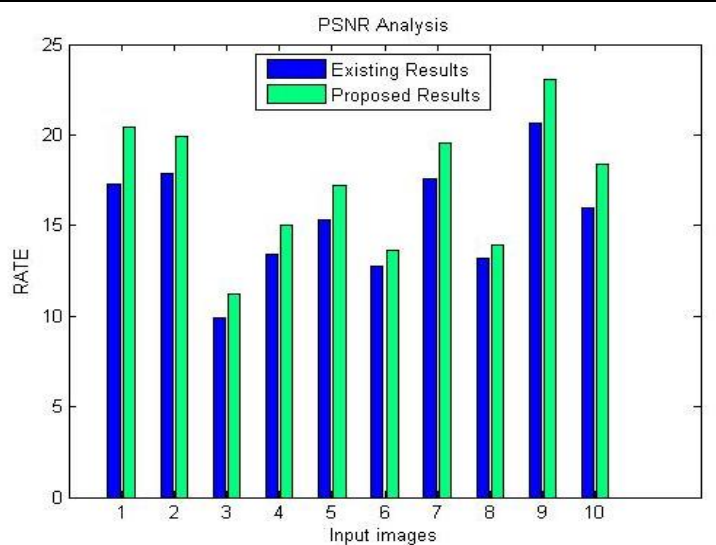

Fig 5: PSNR Analysis

Figure 5 is showing the comparative analysis of the Peak Signal to Noise ratio. Peak Signal to Noise ratio needs to be more for the proposed algorithm for obtaining better results than existing technique. It can be seen clearly in the graph above, the results for proposed algorithm are more in every case as compared to existing technique. This shows the efficiency of proposed algorithm.

\section{ROOT MEAN SQUARE ERROR}

RMSE:- RMSE is the measure of differences between values predicted by a model or an estimator and the values actually observed. Basically, the RMSE represents the sample standard deviation of the differences between input image and the final fog free image. Root mean square error is calculated by the given formulae

$$
\text { RMSE }=\frac{\Sigma\left(\mathrm{f}\left(\mathrm{x}_{\mathrm{i}}\right)-\mathrm{y}_{\mathrm{i}}\right)^{2}}{\mathrm{n}}
$$

Table 3 is showing the comparative analysis of the root mean square error. Table has clearly shown that is less in our case therefore the algorithm has shown significant results over the available algorithm.

\begin{tabular}{|l|l|l|}
\hline \multicolumn{2}{|c}{ Table 3: RMSE Evaluation } \\
\hline & $\begin{array}{l}\text { Existing } \\
\text { Technique }\end{array}$ & $\begin{array}{l}\text { Proposed } \\
\text { Technique }\end{array}$ \\
\hline 1 & 5.0673 & 1.8444 \\
\hline 2 & 6.4641 & 2.0349 \\
\hline 3 & 8.8902 & 2.9731 \\
\hline 4 & 17.182 & 15.742 \\
\hline 5 & 24.307 & 1.8061 \\
\hline 6 & 2.1241 & 2.1241 \\
\hline 7 & 14.792 & 6.8938 \\
\hline 8 & 16.919 & 2.4578 \\
\hline 9 & 8.7066 & 4.8527 \\
\hline 10 & 0.3738 & 0.3738 \\
\hline
\end{tabular}

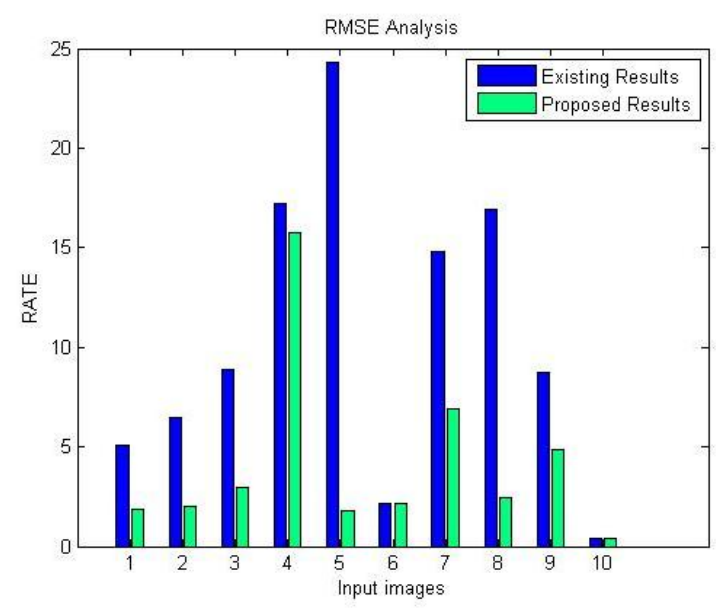

Figure 6: RMSE Evaluation

Figure 6 has shown the quantized analysis of the Root mean squared Error of different images. It is very clear from the plot that there is decrease in RMSE value of images with the use of proposed method over existing method. This decrease represents improvement in the objective quality of the image

Normalized Cross Correlation:- Table 4 has shown the comparison among proposed and the existing strategy based on Normalized Cross Correlation As the Normalized Cross Correlation is more in almost every taken road image; therefore the proposed strategy has shown significant results over the available technique.

Table 4: Normalized Cross Correlation Analysis

\begin{tabular}{|l|l|l|}
\hline IMAGES & $\begin{array}{l}\text { EXISTING } \\
\text { TECHNIQUE }\end{array}$ & $\begin{array}{l}\text { PROPOSED } \\
\text { TECHNIQUE }\end{array}$ \\
\hline 1 & 0.9997 & 0.9998 \\
\hline 2 & 0.9513 & 0.9982 \\
\hline 3 & 0.9553 & 0.9923 \\
\hline 4 & 0.9952 & 0.9952 \\
\hline 5 & 0.9830 & 0.9918 \\
\hline 6 & 0.9985 & 09998 \\
\hline 7 & 0.9992 & 0.9989 \\
\hline 8 & 0.9987 & 0.9997 \\
\hline 9 & 0.9991 & 0.9987 \\
\hline 10 & 0.9973 & 0.9991 \\
\hline
\end{tabular}

Figure 7 has shown the comparison among proposed and the existing strategy based on Normalized Cross Correlation. As the Normalized Cross Correlation is more in almost every taken image; therefore the proposed strategy has shown significant results over the available technique. 


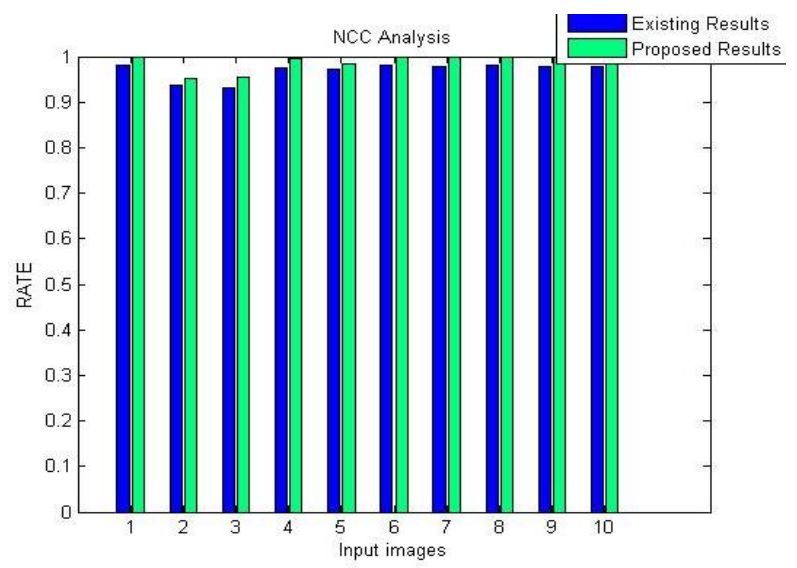

Fig. 7: Normalized Cross Correlation Analysis

\section{CONCLUSION AND FUTURE SCOPE}

In this paper, a new algorithm has been proposed which detects the curved lanes in a very efficient manner. The new algorithm uses fuzzy c means clustering and ant colony optimization to enhance the results of lane detection. The comparison has been made on the basis of various performance metrics. The comparison shows the efficiency of the proposed algorithm over the existing ones.

\section{ACKNOWLEDGMENTS}

I would like to thanks god, my family, my teachers, my friends to guide and support me to write this paper. They always help me when I need.

\section{REFERENCES}

[1] Dietmayer, Klaus, Nico Kaempchen, Kay Fuerstenberg, Joerg Kibbel, Winfried Justus, and Roland Schulz. "Roadway detection and lane detection using multilayer laserscanner." In Advanced Microsystems for Automotive Applications 2005, pp. 197-213. Springer Berlin Heidelberg, 2005.

[2] Rotaru, C., Th Graf, and J. Zhang. "Special Cases of Lane Detection in Construction Areas." In Advanced Microsystems for Automotive Applications 2005, pp. 6169. Springer Berlin Heidelberg, 2005.

[3] Zhou, Yong, Xiaofeng Hu, and Qingtai Ye. "A robust lane detection approach based on MAP estimate and particle swarm optimization." In Computational Intelligence and Security, pp. 804-811. Springer Berlin Heidelberg, 2005.

[4] Möhler, Nikolaus, Dietrich John, and Marina Voigtländer. "Lane detection for a situation adaptive lane keeping support system, the SAFELANE system." InAdvanced Microsystems for Automotive Applications 2006, pp. 485-500. Springer Berlin Heidelberg, 2006.

[5] Jiang, Ruyi, Reinhard Klette, Tobi Vaudrey, and Shigang Wang. "New lane model and distance transform for lane detection and tracking." In Computer Analysis of Images and Patterns, pp. 1044-1052. Springer Berlin Heidelberg, 2009.

[6] Jung, H. G., Y. H. Lee, H. J. Kang, and Jaeyeun Kim. "Sensor fusion-based lane detection for LKS+ ACC system." International journal of automotive technology 10, no. 2 (2009): 219-228.

[7] Romdhane, Nadra Ben, Mohamed Hammami, and Hanene Ben-Abdallah. "A comparative study of vision- based lane detection methods." In Advanced Concepts for Intelligent Vision Systems, pp. 46-57. Springer Berlin Heidelberg, 2011.

[8] Ruyi, Jiang, Klette Reinhard, Vaudrey Tobi, and Wang Shigang. "Lane detection and tracking using a new lane model and distance transform."Machine vision and applications 22, no. 4 (2011): 721-737.

[9] Liu, Lizhuang, Jianzhu Cui, and Jing Li. "Lane Mark Detection Based on Improved Hough Transformation for Vehicle Electronic Technology." InAdvances in Mechanical and Electronic Engineering, pp. 593-598. Springer Berlin Heidelberg, 2012.

[10] Choi, H-C., J-M. Park, W-S. Choi, and S-Y. Oh. "Visionbased fusion of robust lane tracking and forward vehicle detection in a real driving environment."International Journal of Automotive Technology 13, no. 4 (2012): 653 669.

[11] Yao, Wentao, and Zhidong Deng. "Robust Real-Time Lane Marking Detection for Intelligent Vehicles in Urban Environment." In Advances in Automation and Robotics, Vol. 1, pp. 421-428. Springer Berlin Heidelberg, 2012

[12] Moreira, Bruno M., António V. Sousa, Ana M. Mendonça, and Aurélio Campilho. "Automatic lane detection in chromatography images." In Image Analysis and Recognition, pp. 180-187. Springer Berlin Heidelberg, 2012.

[13] Liu, Weirong, and Shutao Li. "An effective lane detection algorithm for structured road in urban." In Intelligent Science and Intelligent Data Engineering, pp. 759-767. Springer Berlin Heidelberg, 2013.

[14] Tao, Junli, Bok-Suk Shin, and Reinhard Klette. "Wrong roadway detection for multi-lane roads." In Computer Analysis of Images and Patterns, pp. 50-58. Springer Berlin Heidelberg, 2013.

[15] Kawatsu, Christopher, Jiaxing Li, and C. J. Chung. "Obstacle \& Lane Detection and Local Path Planning for IGVC Robotic Vehicles Using Stereo Vision." InRobot Intelligence Technology and Applications 2, pp. 667-675. Springer International Publishing, 2014.

[16] Bottazzi, Vitor S., Paulo VK Borges, Bela Stantic, and Jun Jo. "Adaptive regions of interest based on HSV histograms for lane marks detection." InRobot Intelligence Technology and Applications 2, pp. 677-687. Springer International Publishing, 2014.

[17] Hillel, Aharon Bar, Ronen Lerner, Dan Levi, and Guy Raz. "Recent progress in road and lane detection: a survey." Machine Vision and Applications 25, no. 3 (2014): 727-745.

[18] Ran, Feng, Zhoulong Jiang, and Meihua Xu. "VisionBased Lane Detection Algorithm in Urban Traffic Scenes." In Intelligent Computing in Smart Grid and Electrical Vehicles, pp. 409-419. Springer Berlin Heidelberg, 2014

[19] Bottazzi, Vitor S., Jun Jo, Bela Stantic, and Paulo VK Borges. "An Efficient Ego-Lane Detection Model to Avoid False-Positives Detection of Guardrails." InRobot Intelligence Technology and Applications 3, pp. 741-751. Springer International Publishing, 2015. 